\title{
Dynamic precision scaling for low power WCDMA receiver
}

\author{
Hai-Nam Nguyen, Daniel Menard, Olivier Sentieys \\ IRISA/INRIA, University of Rennes, \\ 6 rue de Kerampont \\ F-22300 Lannion \\ Email: hanguyen@irisa.fr
}

\begin{abstract}
One of the most important applications of Digital Signal Processing (DSP) is wireless communication. This kind of application requires low power implementation of DSP, which generally uses fixed-point arithmetic. The fixed-point architectures should be developed to maintain the energy consumption power at a reasonable level. In this paper, an approach which adapts the fixed-point specification according to the input receiver Signal-to-Noise Ratio (SNR) is proposed. To underline our approach interest, the rake receiver of a WCDMA receiver is examined. Results show about $25 \%-40 \%$ energy savings with our dynamic precision approach.
\end{abstract}

\section{INTRODUCTION}

Wireless communication domain is one of the most important sectors for Digital Signal Processing (DSP) applications. In 2007, 74\% of Digital Signal Processors sold were used for wireless applications [1]. Most wireless terminals are nomadic and are supplied by battery. The design of low power terminals is one of the key challenges in this domain. New services are provided (image, video, Internet access) and require high data rate. Consequently, the complexity of the baseband digital part is growing. However, the energy consumption can not be increased due to the limited battery lifetime. Thus, new strategies to reduce or maintain the energy consumption power at a reasonable level must be proposed.

Efficient implementation of DSP applications in embedded systems requires the use of fixed-point arithmetic. Therefore, the vast majority of embedded DSP applications are implemented in fixed-point architectures [2], [3], [4], [5]. Indeed, fixed-point architectures are cheaper and more energy efficient than floating-point architectures because their data word-lengths are lower.

The energy consumption of an application depends on the word-length of the manipulated data. The energy consumption can be reduced by decreasing the data word-length. Nevertheless, this also reduces the computation accuracy and increases the unavoidable error due to finite word-length computation. In [6], an LMS (least mean square) adaptive filter has been studied. The energy consumption is divided by a factor of two between two fixed-point specifications having a Signal to Quantization Noise Ratio (SQNR) of $90 \mathrm{~dB}$ and $30 \mathrm{~dB}$.

The traditional approach to design a fixed-point system is based on the worst-case principle. For a digital communication receiver, the maximal performances and the maximal input dynamic are retained and the more constraint transmission channel is considered. Nevertheless, the noise and the signal levels evolve during time. Now, the data rate depends on the service (video, image, speech) used by the terminal and the required performances (bit error rate) are linked to the service. These various elements show that the fixed-point specification depends on external elements (noise level, input signal dynamic range, quality of service) and can be adapted during time to reduce the average power consumption.

In this paper, an approach in which the fixed-point specification is adapted dynamically according to the input receiver SNR for one modulation scheme and one data rate is proposed. This concept is called Dynamic Precision Scaling (DPS). Our approach interest is shown through a WCDMA (Wide-band Code Division Multiple Access) receiver example.

The paper is organized as follows. In Section II, related works on dynamic word-length adaptation are presented. The principles of Dynamic Precision Scaling is detailed in Section III and the candidate architectures used to implement this concept are also presented. In Section IV, a WCDMA receiver is studied, and especially, the rake receiver module is considered. The energy consumption evolution according to the SNR is analyzed. Finally, Section V draws conclusions.

\section{RELATED WORKS}

In [7], different trade-offs between accuracy and energy are explored in the context of Software Defined Radio. New standards like the further extension of WLAN (802.11n) offer multiple configurations according to link noise robustness and data rate. Different modes (modulation scheme and coding rate) are proposed. For each mode an optimized fixed-point specification is determined and leads to a specific implementation. The selection of a fixed-point specification for each modulation scheme and coding rate makes possible the decrease of the average energy consumption by a factor three. In this approach, the adaptation of the fixed-point specification is only linked to the modulation scheme and coding rate.

In [8], word-length tunable VLSI architecture for an OFDM (Orthogonal Frequency Division Multiplexing) demodulator has been proposed. The data word-length are determined at run time according to the observed error at the system output. For this word-length determination process, word-length search symbols are inserted in the frame. This approach saves $32 \%$ and $24 \%$ of the power consumption for different transmission 


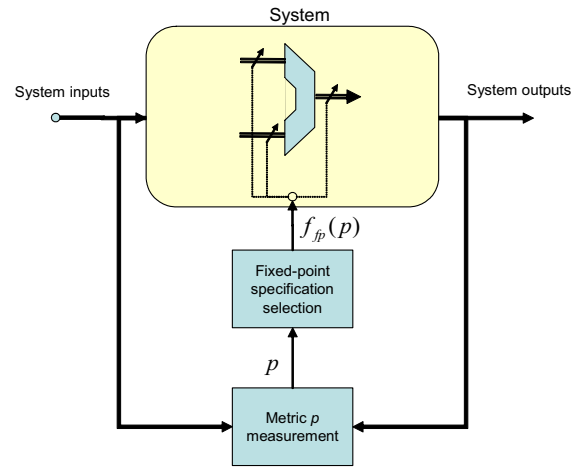

Fig. 1: Synoptic of the Dynamic Precision Scaling approach

channels. This technique requires specific hardware and energy is wasted for the fixed-point optimization process which is carried-out at run time. Moreover, this technique must modify the transmission packet format and can not be used in standard systems.

\section{DyNAmiC PRECISION SCALING (DPS)}

\section{A. Principle}

In the Dynamic Precision Scaling (DPS) approach, to reduce the energy consumption, the fixed-point specification is adapted according to the external environment parameters. During time, the system switches between different fixed-point specifications when the external environment parameters are modified. In our approach, different fixed-point specifications are available. They are determined at the system design level. Let $S_{f p}$, be the set of all the fixed-point specifications which can be used.

To adapt the fixed-point specification to the external environment parameters, a metric $p$ describing the external conditions is used. This metric is determined inside the digital system from the measurement of the input signal and/or the output signal. The fixed-point specification is selected according to this metric value as illustrated in Figure 1.

In the examples presented in this paper, the metric $p$ is the signal to noise ratio (SNR) at the input of the receiver. Different techniques can be used to estimate this SNR [9]. For the WCDMA receiver, data-aided techniques can be used. Indeed, a pilot sequence is available in the control symbol frame $(\mathrm{DPCCH})$ of the WCDMA norm in the context of UMTS/3G wireless communications. Otherwise for the QPSK receiver, SNR estimators can be used with an estimate of the transmitted symbols from the receiver decisions. The selection of the estimator is a trade-off between the estimation quality and the estimator complexity. Indeed, the supplementary energy consumption due to adaptation part must be minimized not to wreck the energy gain due to fixed-point specification adaptation.

\section{B. Architecture for DPS}

To adapt the fixed-point specification during time, the architecture must be programmable or reconfigurable. For processors, a specific code (function) is associated to each fixed-point specification. The processor switches between the parts of code when the metric $p$ is modified. For reconfigurable architectures, a configuration is associated to each fixedpoint specification. The architecture is reconfigured when the metric $p$ is modified. To adapt the fixed-point specification during time the processing unit must be flexible in terms of supported word-length. The aim is to reduce the energy when the word-length is lower than the supported maximal value. Two kinds of approaches are available to minimize the energy consumption through word-length flexibility. One way is to have operators supporting Sub-Word Parallelism (SWP) operations. The operator processes several operations in parallel on operands of smaller word-length. An operator (multiplier, adder, shifter) with a word-length of $N$ is split to execute $k$ operations in parallel on sub-words of $N / k$ wordlength. This technique can accelerate the code execution time up to a factor $k$. The other way is to use operator executing only one operation per cycle but able to manipulate data with different word-lengths. In [10], a multiplier able to perform operations on $9,11,14$ and 16 bits is proposed.

\section{WCDMA RECEIVER}

WCDMA is a standard for the third-generation of cellular network which is based on DS-CDMA (Direct Spread CDMA) technology. In these systems, a rake receiver is used to benefit from the effects of multi-path fading. A finger is allocated to each path to decode the symbol associated with the path. One important component associated with the rake receiver is the path searcher. A path searcher finds the delay of the different paths, which is then used to synchronize the input signal with the code generated in the receiver and thus to obtain an optimal combination of received energy.

In WCDMA, two layers of spreading codes are used [11]: channelization code and scrambling code. The channelization code $C_{c h}$ is used to achieve orthogonality between channels when time-shift is equal to 0 . The scrambling codes used in uplink are Gold codes $S_{G}$. The input data $d_{t}$ is multiplied with the spreading codes, and the transmitted signal $\mathrm{TX}_{t}$ is equal to $\mathrm{TX}_{t}=d_{t} C_{c h} S_{G}$. In a multi-path (time dispersive) Rayleigh channel, the global received signal $s(n)$ is the sum of elementary signal $s_{i n}^{k}(n)$ for different channel paths. Let $\tau_{i}$ and $\alpha_{i}$ be respectively the delay and the complex amplitude of $k^{\text {th }}$ path in the channel. The global received signal $s(n)$ expression is equal to

$$
s(n)=\sum_{i} s_{i n}^{k}(n)+n_{i n}(n)=\sum_{i} \alpha_{i} \operatorname{Tx}\left(n-\tau_{i}\right)+n_{i n}(n)
$$

The term $n_{i n}(n)$ represents the noise term and is made-up of the receiver thermal noise and the interference of the other users. This term can be considered gaussian with variance $\sigma^{2}$. Assuming that a user has one DPDCH channel, $d_{t} C_{c h}$ take values in $\{ \pm 1 \pm i\}$. Thus $\mathrm{TX} \in\{ \pm 2, \pm 2 i\}$. In our simulations, TX is normalized into $\{ \pm 1, \pm i\}$, hence its power is equal to one. By definition, $\mathrm{SNR}=2 E_{b} / N_{0}=1 / 2 \sigma^{2}$. The increase 


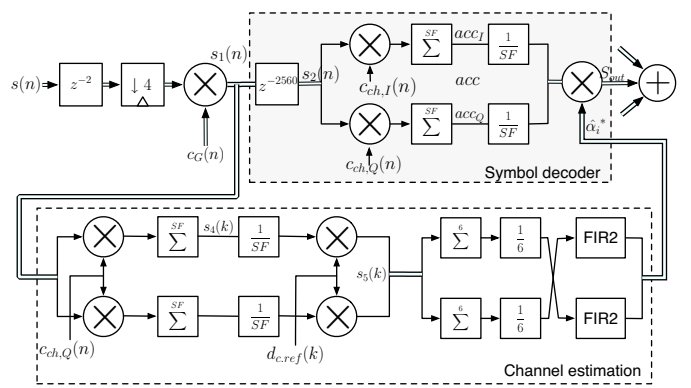

Fig. 2: Data flow graph of the $i^{t h}$ finger of the WCDMA rake receiver.

of the number of users in a cell rises the interferences. These interferences are processed as noises and thus decrease the SNR. To take account of the different cases (a few number of communications in a cell with good transmission conditions and a great number of users with bad transmission conditions) a great range of SNR is considered (0 to $25 \mathrm{~dB}$ ).

\section{A. Range estimation}

The first step of the fixed-point conversion process corresponds to the determination of the data integer part wordlength. This step requires to determine each data dynamic range. An analytical approach based on interval arithmetic [12] is used to estimate the dynamic and to guarantee no overflow. The flow graph of one rake receiver finger is presented in Figure 2. This system is made-up of two modules corresponding to the channel estimation and to the symbol decoding. The first one computes the complex amplitude $\alpha_{i}$ of the path and the second one estimates the transmitted symbol $\widehat{s}_{\text {out }}$. In the finger, the correlation between the input signal and the codes is used to amplify useful signal $s_{i n}^{k}(n)$ to detect the transmitted symbol. The correlation process increases the range of the useful signal, but not that of the receiver noise $n_{\text {in }}(n)$. From this property, an approach is then proposed to determine more accurately the data dynamic range. Before the correlation process, the whole useful signal $s_{i n}^{k}(n)$ plus noise $n_{i n}(n)$ is considered. After this process, only the useful signal $s_{i n}^{k}(n)$ is taken into account when calculating the dynamic range.

The input signal $s(n)$ consisting of desired signal $s_{i n}^{k}(n)$ plus noise $n_{i n}(n)$ is normalized to have the maximum amplitude of both real and imaginary parts equal to one. Because a gaussian noise $\sigma^{2}$ has $99.7 \%$ of its values in $[-3 \sigma, 3 \sigma]$, assuming the real and imaginary parts of $\alpha_{i} \mathrm{TX}-\tau_{i}$ are in $[-1,1]$, the input $s(n)$ is considered in $[-1-3 \sigma, 1+3 \sigma]$. The normalization process is thus implemented by dividing the input $s(n)$ by $1+3 \sigma$ then cut off by 1 . The expressions of the dynamic range for the accumulator output $\left(a c c_{I}\right)$ and the finger output $s_{\text {out }}$ are equal to

$$
\max \left(\mid \text { acc }_{I} \mid\right)=\frac{4 . S F}{1+3 \sigma} \quad \max \left(\left|s_{\text {out }}\right|\right)=\frac{4}{(1+3 \sigma)^{2}}
$$

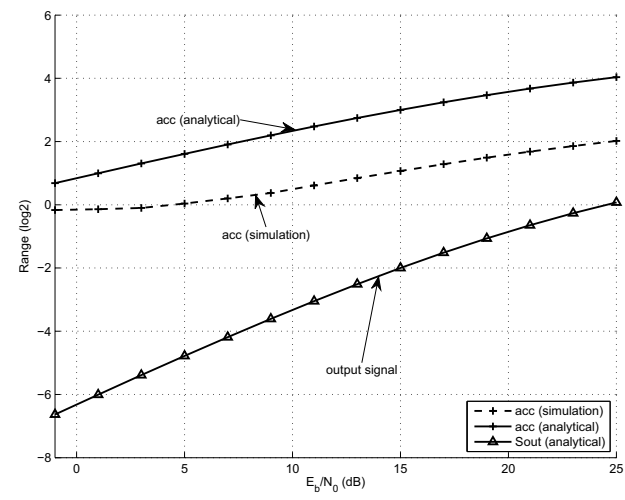

Fig. 3: Estimated and simulation based values of data range in different channel conditions for the symbol decoder.

The estimation values based on analytical approach are presented in Figure 3 and compared with those based on simulations. In both simulation and estimation, for the accumulation output, there is a difference of 3 bits between $0 \mathrm{~dB}$ and $25 \mathrm{~dB}$. For the finger output, there is a difference of 4 bits between $0 \mathrm{~dB}$ and $15 \mathrm{~dB}$ and of 6 bits between $0 \mathrm{~dB}$ and $25 \mathrm{~dB}$. It is noticed that from one to two bits differ between estimated and simulated results. Nevertheless, the evolutions of the dynamic range according to the SNR are identical for analytical and simulation based estimations. This confirms the validity of our approach to estimate the dynamic range in the WCDMA receiver. The difference between the two lines can be explained by the channel model used in the simulation. If a single path channel model, for example, is used, the difference is less than 1 bit. Moreover, the analytical estimations are known to be more pessimistic.

\section{B. Energy optimization under accuracy constraint}

The second step of the fixed-point conversion process corresponds to the determination of the fractional word-length. The use of finite precision arithmetic conducts to an unavoidable quantization error $n_{q}(n)$ which degrades the computation accuracy. The data word-length rising decreases the quantization noise power but increases the energy consumption. The computation quality is analyzed through the quantization noise power $P_{n_{q}}$. A minimal computation accuracy must be provided to guarantee that the system performances are maintained. For the symbol decoding module, the performances are evaluated with the bit-error rate (BER) so that the use of finite precision does not modify the reference infinite precision BER of $a \%$.

Thus, the aim of this step is to minimize the energy consumption $E$ under an accuracy constraint $P_{n_{q}}^{\max }$. The technique presented in [13] is used to determine the accuracy constraint. This technique is based on a floating-point precision and the fixed-point behavior is modeled by a single noise source located at the system output.

The accuracy constraint has been determined for different SNR values. The results are presented in Figure 4. The line $P_{s_{\text {out }}}$ and $P_{n_{\text {out }}}$ correspond to the level (power) respectively of 


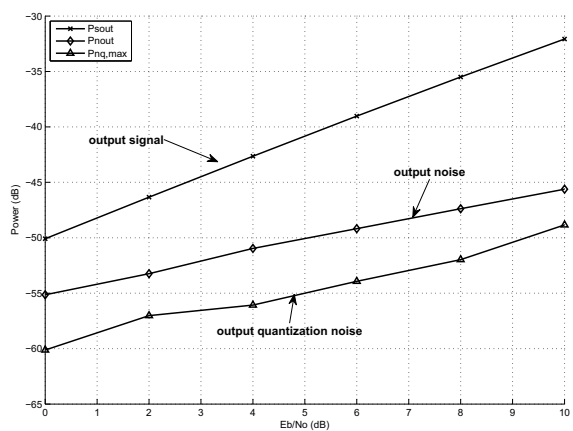

Fig. 4: Signal and noise power levels according to the SNR

the desired signal $s_{\text {out }}$ (symbol) and the receiver noise $n_{\text {out }}$ at the system output. The difference between the lines $P_{s_{\text {out }}}$ and $P_{n_{\text {out }}}$ corresponds to the output signal to noise ratio (SNR). The difference between the lines $P_{s_{\text {out }}}$ and $P_{n_{q}}$ corresponds to the output signal to quantization noise ratio (SQNR).

The results show that to decrease the BER when the SNR increases, the SQNR must be increased. More accuracy is required to reduce the decision errors due to finite precision arithmetic. The difference between the lines $P_{n_{\text {out }}}$ and $P_{n_{q}}^{\max }$ is practically constant. In fixed-point, to maintain a BER close to the one for infinite precision, the quantization noise must be proportional to the receiver noise. The maximal value of the quantization noise (accuracy constraint) is equal to the receiver noise minus $4 \mathrm{~dB}$. These results show that in a communication receiver, the accuracy constraint can be directly obtained from the receiver noise level.

After the determination of the maximal quantization noise power (accuracy constraint), the data word-lengths are optimized. The energy consumption is minimized under the accuracy constraint. The cost function corresponding to the energy consumption is estimated from the sum of the energy of the different operations involved in this processing (arithmetic operation, register and memory transfer). The energy model used in this paper is based on a database where each operator is characterized for the various word-lengths [6]. The technique and the algorithm used to minimize the energy consumption under accuracy constraint are presented in [6].

Simulation results are presented in Figure 5. Since the word-length of different operators takes discret values, the energy consumption function has the form of a step function. A specific fixed-point configuration is associated with each SNR interval where the energy consumption is constant. Optimization results show that, for a SNR varying from 0 $\mathrm{dB}$ to $10 \mathrm{~dB}, 25 \%$ of energy consumption can be saved. For a SNR varying from $0 \mathrm{~dB}$ to $25 \mathrm{~dB}$, up to $40 \%$ of energy consumption can be saved on applying our dynamic precision approach.

\section{CONCLUSion}

This paper has addressed the concept of energy consumption reduction by adapting the fixed-point specification. The

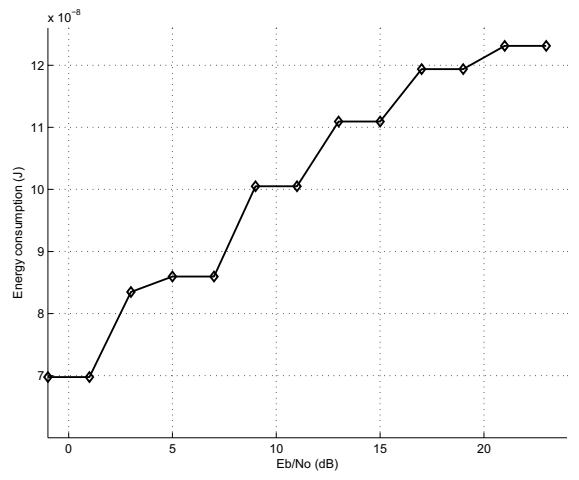

Fig. 5: Energy consumption according to the SNR

concept and the target architecture were presented. We have shown that the energy consumption increases when the SNR increases. By applying the dynamic precision scaling, up to $40 \%$ of consumed energy can be saved for the finger of a WCDMA receiver. The same approach has been conducted for the searcher module. The performances are evaluated through the mis-detection and false-alarm probabilities. The results for the searcher show that the DPS approach allows reducing up to $25 \%$ the energy consumption.

\section{REFERENCES}

[1] W. Strauss, "Hanging up on analog and flexing Wireless/DSP muscles," Forward Concepts, Tech. Rep., 2008.

[2] B. Evans, "Modem Design, Implementation, and Testing Using NI's LabVIEW," in National Instrument Academic Day, Beirut, Lebanon, June 2005.

[3] J. Eyre and J. Bier, "The evolution of DSP processors," IEEE Signal Processing Magazine, vol. 17, no. 2, pp. 43-51, March 2000.

[4] W. Strauss, "DSP chips take on many forms," DSP-FPGA.com Magazine, March 2006.

[5] J. Eyre and J. Bier, "DSPs court the consumer," IEEE Spectrum, vol. 36, no. 3, pp. 47-53, 1999.

[6] R. Rocher, D. Menard, N. Herve, and O. Sentieys, "Fixed-Point Configurable Hardware Components," EURASIP Journal on Embedded Systems, vol. 2006, no. 1, pp. 20-20, 2006.

[7] D. Novo, B. Bougard, A. Lambrechts, L. Van der Perre, and F. Catthoor, "Scenario-based fixed-point data format refinement to enable energyscalable software defined radios," Design, Automation and Test in Europe, 2008 (DATE '08), pp. 722-727, March 2008.

[8] S. Yoshizawa and Y. Miyanaga, "Tunable word length architecture for low power wireless OFDM demodulator," Circuits and Systems, 2006. ISCAS 2006. Proceedings. 2006 IEEE International Symposium on, May 2006.

[9] D. Pauluzzi and N. Beaulieu, "A comparison of SNR estimation techniques for the AWGN channel," Communications, IEEE Transactions on, vol. 48, no. 10, pp. 1681-1691, Oct 2000.

[10] M. Bhardwaj, R. Min, and A. Chandrakasan, "Power-aware systems," in 34th Asilomar Conference on Signals, Systems and Computers, December 2000.

[11] K. Tachikawa, W-CDMA Mobile Communications System. Wiley, 2002.

[12] R. Kearfott, "Interval Computations: Introduction, Uses, and Resources," Euromath Bulletin, vol. 2, no. 1, pp. 95-112, 1996.

[13] D. Menard, R. Rocher, O. Sentieys, and O. Serizel, "Accuracy Constraint Determination in Fixed-Point System Design," EURASIP Journal on Embedded Systems, Accepted for publication in 2008. 